\title{
Evaluating fear of hypoglycemia, pediatric parenting stress, and self-efficacy among parents of children with type 1 diabetes and their correlation with glycemic control
}

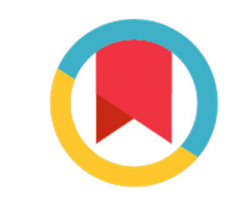

\author{
Fatemehsadat Amiri ${ }^{1}$, Mohammadreza Vafa*1, Linda Gonder-Frederick ${ }^{2}$, Karen Vajda ${ }^{2}$, Mohammadebrahim Khamseh ${ }^{3}$, \\ Alireza Abadi $^{4}$, Amin Salehpour ${ }^{1,5}$
}

Received: 5 Nov 2017

Published: 30 Nov 2018

\begin{abstract}
Background: This study was designed to determine the level of fear of hypoglycemia ( $\mathrm{FoH})$, pediatric parenting stress and selfefficacy in parents of children with type 1 diabetes (T1D).

Methods: In this cross-sectional study, 61 families of children with T1D who had been diagnosed for at least 6 months recruited from "Gabric Diabetes Education Association" in Tehran. Sixty mothers and 41 fathers of 61 children (26 girls, age: 6.0-12.7 years) were assessed using the Hypoglycemia Fear Survey-Parent (HFS-P), Pediatric Inventory for Parents (PIP) and Self-Efficacy for Diabetes Scale-Parent (SED-P) questionnaires. Pearson correlation analysis was used to compute the correlation between HFS-P, PIP and SED$\mathrm{P}$ scores separately for mother and fathers.

Results: Only $8.3 \%$ of children had controlled diabetes. Internal reliability of the Persian version of all questionnaires was good. FoH were higher for mothers. Mothers whose children had diabetes for less than two years had significantly lower mean HFS-Behavior subscale (HFS-B) scores than mothers whose children had diabetes for more than two years. There was a positive correlation between fathers' mean HFS-B score and children's total insulin dose per day. Parents' FoH score was positively correlated with increased pediatric parenting stress. Findings also showed considerable emotional distress in $51 \%$ of mothers and $29.7 \%$ of fathers. Frequency of selfmonitoring blood glucose tests (SMBG) correlated negatively with HbA1c.

Conclusion: We concluded that parents with high levels of $\mathrm{FoH}$ and stress may benefit from diabetes education. Important implications for education are considering psychological adjustment, recognizing diabetes-related fear and stress in parents, encouraging fathers to become actively involved in the child's diabetes management and emphasizing the importance of SMBG.
\end{abstract}

Keywords: Hypoglycemia, Fear, Parenting stress, Self-efficacy, Type 1 diabetes

Conflicts of Interest: None declared

Funding: Vice Chancellor of Research, Iran University of Medical Sciences, Tehran, Iran

*This work has been published under CC BY-NC-SA 1.0 license.

Copyright $\subseteq$ Iran University of Medical Sciences

Cite this article as: Amiri F, Vafa M, Gonder-Frederick L, Vajda K, Khamseh M, Abadi A, Salehpour A. Evaluating fear of hypoglycemia, pediatric parenting stress, and self-efficacy among parents of children with type 1 diabetes and their correlation with glycemic control. Med J Islam Repub Iran. 2018 (30 Nov);32:119. https://doi.org/10.14196/mjiri.32.119

\section{Introduction}

Type 1 diabetes (T1D) is the most common chronic metabolic disorder in children, accounts for $5-10 \%$ of the total

Corresponding author: DrMohammadreza Vafa, vafa.m@iums.ac.ir

1. Department of Nutrition, School of Public Health, Iran University of Medical Sciences, Tehran, Iran

2. Department of Psychiatry and Neurobehavioral Sciences, University of Virginia, Virginia, USA

3. Endocrine Research Center, Institute of Endocrinology and Metabolism, Iran University of Medical Sciences, Tehran, Iran

4. Social Determinants of Health Research Center, Shahid Beheshti University of Medical Sciences, Tehran, Iran

5. Occupational Health Research Center, Iran University of Medical Sciences, Tehran, Iran cases of diabetes worldwide. According to the last International Diabetes Atlas report, more than 500,000 children

$\uparrow$ What is “already known” in this topic:

Previous studies have shown that there are numerous psychosocial and behavioral factors that can affect children's glycemic control in family framework including parental fear about their child's hypoglycemia, stress and self-efficacy. However, to the best of our knowledge, there has been no previous research in this field in Iran.

\section{$\rightarrow$ What this article adds:}

This study demonstrated that the Persian version of the HFS-P, PIP, and SED-P are valid and reliable instruments to measure parental fear, stress and self-efficacy in managing their children's diabetes and these questionnaires could be used to identify those families who are at risk. 
developed T1D in 2015 and is suggested to increase by $3 \%$ per year worldwide (1). There is limited information about glycemic control, management, and prevalence of diabetic complications in children from Asia (2, 3). Diabetes management and complications in patients from 230 centers in Asia were reported in "Diabcare-Asia". Only 152 (0.7\%) were younger than 18 years, and the mean glycated hemoglobin $(\mathrm{HbA} 1 \mathrm{c})$ level was higher in this younger subgroup $(10.7 \% \pm 3.0 \%)$ in comparison with the whole cohort $(8.6 \% \pm 2.2 \%)(2)$. Dealing with diabetes in children can be challenging, and there are numerous psychosocial and behavioral factors that can affect day-to-day treatment in children. Specially, in children who cannot recognize and verbalize symptoms of hypoglycemia or hyperglycemia, their unpredictable dietary behaviors and physical activity levels make diabetes more difficult to be managed. The International Society for Pediatric and Adolescent Diabetes (ISPAD) Consensus Guidelines stated that diabetes management could be affected by psychosocial factors as the most important influences (4). We hypothesized that there are some factors that affect children's glycemic control in family framework including (a) parental fear about their child's hypoglycemia, (b) parental stress associated with caring for a child with diabetes, (c) parental beliefs about their ability to manage their child's illness (self-efficacy) (5).

Hypoglycemia is an important limiting factor for glycemic control and causes symptoms which may frighten both children and their parents. There is evidence that fear of hypoglycemia $(\mathrm{FoH})$ may be a significant barrier to diabetes management, quality of life and future health outcomes (68 ). Although studies have shown that new insulin regimen and insulin analogs may lower the risk and frequency of hypoglycemic episodes, hypoglycemia remains a problem in children with T1D $(9,10)$.

Pediatric parenting stresses experienced by parents of children with chronic illness like diabetes are unique compared to general parenting and caregiver stress (11). A large body of pediatric psychology research has focused on mothers, leaving the fathers' experiences largely unexamined $(12,13)$. Those studies examining both parents' adjustments to children's diabetes have reported varied findings (12); however, one study found that mothers had higher rates of post-traumatic stress than fathers (14). Parents of children with poor diabetes control receiving conventional insulin regimens also report more stress (5). Highest level of parental stress has been reported during the weeks immediately after a child's diagnosis, which then declines but does not certainly diminish completely over the subsequent year (4).

Parental efficacy in managing their child's diabetes is negatively related to pediatric parenting stress (5). In asthmatic children, lower parental self-efficacy has been associated with increased asthma-related morbidity (15). Researches on self-efficacy within the diabetes literature are limited $(5,16,17)$.

The present study aimed to describe glycemic control and investigate factors that we hypothesized to be associated with, including FoH, emotional distress and self-efficacy in managing diabetes between mothers and fathers of children with T1D. Also, this study was designed to conduct a psychometric investigation and to test the reliability and validity of a Persian version of the Hypoglycemia Fear SurveyParent version (HFS-P), Pediatric Inventory for Parents (PIP) and Self-Efficacy for Diabetes Scale- Parent version (SED-P) to assess whether these questionnaires can be useful tools for assessment of $\mathrm{FoH}$, parenting stress and selfconfidence in managing children's diabetes (respectively). Moreover, this study examined parent report of the above parameters in a sample of children with T1D with these tools. Another purpose was to evaluate associations of the HFS-P, PIP, and SED-P with children's glycemic control.

\section{Methods}

\section{Participants and Procedure}

The study design was cross-sectional and was approved by the Medical Ethical Committee of Iran University of Medical Sciences. Families were identified through a review of the "Gabric Diabetes Education Association" (member of the International Diabetes Federation) database (www.gabric.ir), and then contacted by a member of the research team. Parents were eligible to participate if had a child 6-12 years' old who had been diagnosed with T1D for at least 6 months. Families were excluded if the child suffered from another disease known to affect growth or another autoimmune disease (e.g. thyroid, celiac). A total of 75 families were approached for the study, and 61 agreed to participate and were eligible ( $81.33 \%$ recruitment rate). Parents and children came to the offices of the Gabric Diabetes Education Association (GDEA), where they provided written informed consent, then completed a battery of questionnaires, including HFS-P, additional psychological questionnaire including PIP to assess parental stress and SED-P. They also completed a diabetes history questionnaire, including an item to assess the frequency of severe hypoglycemic episodes in the last 3 months. Severe hypoglycemia was defined as children's hypoglycemia with unconsciousness during which blood glucose was so low that self-treatment was not possible because of mental confusion and external assistance was required. Children's glycemic control was examined by $\mathrm{HbA} 1 \mathrm{c}$ level which was analyzed on whole blood collected in EDTA vacutainer tubes and measured by a designated high-performance liquid chromatography (HPLC) method (Tosoh G7 Automated HPLC Analyzer; Tosoh Bioscience, Grove City, OH, USA). Blood samples for HbA1c analysis were taken on the same day when questionnaires were completed at a laboratory.

\section{Measures and Questionnaires}

Questionnaires were translated into Persian for this study using the translation process recommended by the World Health Organization (http:/www.who.int/ substance abuse/research_tools/translation/en), which includes forward and backward translation, pre-testing, and cognitive debriefing.

Hypoglycemia Fear: We used the HFS-P to assess parents' worries and behaviors related to hypoglycemia (18). HFS-P is a 25 -item survey that includes a 15 -item Worry subscale (HFS-W) and a 10-item Behavior subscale (HFS- 
B). The Worry subscale items measure anxiety-provoking aspects of hypoglycemia and the items in the Behavior subscale measure specific behaviors aimed at avoiding hypoglycemia (18). This questionnaire uses a Likert response format ranging from 1 (never) to 5 (always). The HFS-P subscale scores and the total score are obtained by summing the items for respectively the Worry subscale (range 1575), the Behavior subscale (range 10-50) and the HFS-P total (range 25-125) (7). Other translations of the HFS-P, including English and Norwegian, have previously demonstrated adequate reliability and validity $(7,19)$. Higher scores indicate higher $\mathrm{FoH}$. In accordance with original HFS-P items, we asked the parents some questions about the frequency of problematic hypoglycemic (refer to the children's hypoglycemia with consciousness but need parent's help) episodes during the past 3 months.

Pediatric parenting stress: Parenting distress and stress were assessed using the PIP which measures experiences related to parenting a child with a medical condition (11). The PIP asks parents to rate the frequency and difficulty of 42 events usually reported by parents of children with a chronic illness. The subscales are communications (with child, partner, or healthcare team in 9 items), emotional distress (impact of illness on sleeping quality and mood in 15 items), medical care (carrying out medical regimen in 8 items) and role function (impact of disease on ability to work and caring for other children in 10 items). Responses are scored using two 5-point Likert scales ranging from 'never' to 'very often' (frequency scale) and 'not at all' to 'extremely' (difficulty scale). The PIP is scored separately for each of the four domains. There are also two overall total scores comprised of the sum for each of the four domains, yielding total $\mathrm{F}$ and total D scores (range: 42-210) to reflect the frequency of stressful events (PIP-F), and the amount of difficulty experienced by parents in managing these events (PIP-D). The PIP has demonstrated strong internal consistency and constructs validity when utilized with parents of children with diabetes (5). Higher scores indicate more frequent problems/more difficulty and increased pediatric parenting stress.

Parent confidence: Parent confidence in managing their child's diabetes was assessed using the SED-P (20). The
SED-P is a 22-item self-report survey that assesses respondents' confidence in performing daily diabetes management tasks. Participants respond using a 5- point Likert scale ranging from 'very sure I cannot' to 'very sure I can'. Higher scores indicate higher self-efficacy in managing their child's diabetes.

\section{Statistical Analysis}

Descriptive statistics, correlations, group comparisons, and Cronbach's alpha reliability coefficient were performed using SPSS 22 (SPSS Inc., Chicago, IL, USA). A p-value less than 0.05 was considered statistically significant. Sample characteristics and HFS-P, PIP and SED-P scores were explained using descriptive statistics including means, standard deviations (SD), and frequency distributions. We used Pearson correlation analysis to compute the correlation between HFS-P, PIP and SED-P scores separately for mother and fathers. Because of the importance of age and duration of diabetes in children, all of the analyses compared across age ( $\leq 9$ and $\geq 10$ years) and diabetes duration categories ( $<2$ and $\geq 2$ years) as well.

\section{Results}

Internal consistencies of the HFS-P, PIP, and SED-P were calculated using Cronbach's alpha coefficient for the total scale and each subscale. Cronbach's alpha for the Persian version of the HFS-P showed good internal consistency for the HFS-W subscale (mothers 0.89; fathers 0.89), the HFS-B subscale (mothers 0.86; fathers 0.86) and the HFS-P total (mothers 0.94; fathers 0.94). The large degree of accordance between the Cronbach's alpha values for mothers and fathers reinforces the reliability of the Iranian HFS-P. Results found alpha coefficients of 0.93 in both mothers and fathers for the PIP-total frequency domain, 0.96 and 0.95 respectively in mothers and fathers, for PIP-total difficulty domain suggesting the excellent internal consistency for the Persian version PIP. Also, results found alpha coefficients of 0.74 in both mothers and fathers for the SED-P questionnaire.

Parents of 61 children (60 mothers and 45 fathers) completed the questionnaires. Mothers were between 25 and 49 years of age (36.2 \pm 5.6$)$, the majority were high school

Table 1. Children's demographic and clinical characteristics

\begin{tabular}{|c|c|c|c|}
\hline Variable & $\mathrm{n}(\%)$ & Mean (range) & SD \\
\hline$\overline{\text { Age (years) }}$ & 61 & $9.2(6.0-12.7)$ & 2.0 \\
\hline Boys & $35(57.4)$ & & \\
\hline $\mathrm{HbA} 1 \mathrm{c}$ & 60 & $9.4(6.1-13.7)$ & 1.8 \\
\hline - $\quad$ Good Control $(<7.5 \%)$ & $5(8.3)$ & & \\
\hline - $\quad$ Poor Control $(\geq 7.5 \%)$ & $55(91.7)$ & & \\
\hline Duration of diabetes (months) & 61 & $38.7(6.0-126.0)$ & 24.5 \\
\hline Total insulin dose per day (units) & 61 & $28.3(9.0-80.0)$ & 14.6 \\
\hline Insulin type & 61 & & \\
\hline • Human & $52(85.2)$ & & \\
\hline - Analogue & $9(14.8)$ & & \\
\hline Number of Insulin Injections & 61 & & \\
\hline$\bullet \quad \leq 3 /$ day & $50(82)$ & & \\
\hline - $\quad \geq 4 /$ day & $11(18)$ & & \\
\hline BG Monitoring Frequency per day & 58 & & \\
\hline - $\quad \leq 3$ times/day & $33(56.9)$ & & \\
\hline - $\quad \geq 4$ times/day & $25(43.1)$ & & \\
\hline Hypoglycemia (past 3 months)* & 59 & $1.4(0-36)$ & 5.4 \\
\hline
\end{tabular}


graduates $(65.6 \%)$ and $75 \%$ did not work outside the home. Fathers were between 30 and 58 years of age (42.0 \pm 5.9$)$, and the majority were high school graduates (64\%), employed $(94.8 \%)$ and reported monthly family income under $\$ 800(88.5 \%)$.

All parents reported being of Iranian nationality. Table 1 presents the demographic and clinical characteristics of the 61 children. Mean \pm SD HbA1c was $9.4 \pm 1.76$ (range: 6.1 13.7 ) and only $8.3 \%$ of children had controlled diabetes by recommended standards (HbA1c $<7.5 \%$ (22)). Moreover, HbAlc values was higher in children 10 and older $(9.9 \pm 1.8)$ compared to children 9 and younger $(9.0 \pm 1.7), \mathrm{F}(1,58)=$ $3.79, \mathrm{p}=0.05$. There was no significant association between HbAlc and other variables including HFS-P, PIP, and SED-P.

Table 2 summarizes the mean (SD) scores for the HFS$\mathrm{P}, \mathrm{PIP}$ and SED-P measures. HFS-P total scores were higher for mothers $(51.7 \pm 15.2)$ than fathers $(44.8 \pm 14)$, $\mathrm{t}=2.39, \mathrm{p}=0.022$. Mothers whose children had diabetes for $<2$ years, had significantly lower mean HFS-Behavior subscale (HFS-B) scores than mothers whose children had diabetes for $>2$ years $F(1,58)=7.50, p=0.008$. There was a positive correlation between fathers' mean HFS-B score and children's total insulin dose per day $(\mathrm{r}=0.30, \mathrm{p}=0.044)$. PIP scores also differed between mothers and fathers. The mean PIP scores were $128.0 \pm 16.4$ (frequency) and $120.9 \pm 28.7$ (difficulty) for mothers and $113.1 \pm 19.1$ (frequency) and 108.2 \pm 31.3 (difficulty) for fathers. PIP scores in $51 \%$ of the mothers and $29.7 \%$ of the fathers were above the frequency midpoint cut-off $\geq 126$, and $51 \%$ of the mothers and $40 \%$ of the fathers had scores above the difficulty midpoint cut-off $\geq 126$ indicating considerable symptomatic emotional distress. SED-P scores did not significantly differ between mothers $(79.9 \pm 10.8)$ and fathers (80.5 \pm 11.2$)$. SED-P scores were above the suggested midpoint cut-off level of 66 in near all mothers and fathers, indicating acceptable self-efficacy in managing their child's diabetes. Correlational analyses indicated no significant association between SED-P and parents' FoH or PIP.

Table 3 summarizes validity analyses. In mothers, both the HFS-W subscale and HFS-P total scores correlated positively with several PIP scores. HFS-W subscale and HFS-P total correlated negatively with PIP-medical care (frequency). Moreover in fathers, HFS-W subscale correlated positively with measures of parenting stress, including PIP-emotional distress (frequency and difficulty), PIPmedical care (difficulty), PIP-role function (frequency and difficulty), PIP-total (frequency and difficulty) and HFS-P total correlated positively with measures of PIP-emotional distress (frequency), PIP-medical care (difficulty), PIP-role function (difficulty), PIP-total (difficulty).

No significant associations emerged between parents' FoH, pediatric parenting stress or self-efficacy and demographic characteristics (age, education, employment, and income). There were no significant associations between parents' FoH, episodes of children's hypoglycemia in the

Table 2. Mean Hypoglycemia Fear Survey-Parent (HFS), Pediatric Inventory for Parents (PIP) scores and Self-Efficacy for Diabetes Scale- Parent (SED-P) in mothers and fathers of children with Type 1 diabetes (aged 6-12.7 years)

\begin{tabular}{|c|c|c|c|c|c|c|}
\hline \multirow[t]{2}{*}{ Score } & \multicolumn{3}{|c|}{ Mothers } & \multicolumn{3}{|c|}{ Fathers } \\
\hline & $\mathrm{n}$ & Mean (range) & SD & $\mathrm{n}$ & Mean (range) & SD \\
\hline HFS-B subscale & 60 & $26.9(15-41)$ & 6.9 & 45 & $25.3(13-42)$ & 7.3 \\
\hline HFS-W subscale & 60 & $24.8(10-50)$ & 12.1 & 44 & $19.7(1-59)$ & 11.8 \\
\hline HFS-P Total & 60 & $51.7(25-100)^{*}$ & 15.2 & 44 & $44.84(17-86)^{*}$ & 14.0 \\
\hline PIP- Communication frequency & 57 & $24.4(16-34)$ & 4.1 & 39 & $22.6(13-31)$ & 4.7 \\
\hline PIP- Emotional distress frequency & 56 & $48.2(26-68)$ & 8.6 & 37 & $40.8(22-59)$ & 9.4 \\
\hline PIP- Medical care frequency & 56 & $30.9(23-40)$ & 3.2 & 38 & $26.9(17-33)$ & 4.3 \\
\hline PIP- Role function frequency & 55 & $24.5(14-40)$ & 5.3 & 37 & $22.9(14-33)$ & 5.4 \\
\hline PIP- Total frequency & 56 & $128.0(98-172)^{*}$ & 16.4 & 37 & $113.1(73-151)^{*}$ & 19.1 \\
\hline PIP- Communication difficulty & 57 & $23.6(8-40)$ & 7.1 & 37 & $19.9(8-32)$ & 6.9 \\
\hline PIP- Emotional distress difficulty & 55 & $52.1(28-74)$ & 10.9 & 35 & $46.4(15-69)$ & 14.3 \\
\hline PIP- Medical care difficulty & 56 & $19.5(8-33)$ & 6.7 & 36 & $16.8(8-29)$ & 5.9 \\
\hline PIP- Role function difficulty & 55 & $26.4(10-46)$ & 8.2 & 35 & $24.9(10-38)$ & 7.7 \\
\hline PIP- Total difficulty & 56 & $120.9(64-186)^{*}$ & 28.7 & 35 & $108.2(42-152)^{*}$ & 31.3 \\
\hline SED-P & 61 & $79.9(57-111)$ & 10.8 & 42 & $80.5(54-110)$ & 11.2 \\
\hline
\end{tabular}

Table 3. Correlations between Hypoglycemia Fear Survey-Parent version (HFS-P) and Pediatric Parenting Stress (PIP) scores in mothers and fathers of children (aged 6-12.7 years) with Type 1 diabetes

\begin{tabular}{|c|c|c|c|c|c|c|}
\hline \multirow[t]{2}{*}{ PIP Score } & \multicolumn{3}{|c|}{ Mother } & \multicolumn{3}{|c|}{ Father } \\
\hline & $\mathrm{n}$ & HFS-W subscale & HFS-P total & $\mathrm{n}$ & HFS-W subscale & HFS-P total \\
\hline \multicolumn{7}{|l|}{ Stress frequency: } \\
\hline - PIP-Communication & 57 & $0.339 * *$ & $0.312 * *$ & 39 & - & - \\
\hline - $\quad$ PIP- Emotional distress & 56 & $0.425^{*}$ & $0.440 *$ & 37 & $0.520^{*}$ & $0.346^{* * *}$ \\
\hline - $\quad$ PIP- Medical care & 56 & $-0.302 * * *$ & $-0.275 * * *$ & 38 & - & - \\
\hline - $\quad$ PIP- Role function & 55 & $0.278 * * *$ & $0.315 * *$ & 37 & $0.377 * * *$ & - \\
\hline - PIP- Total & 56 & $0.326^{* *}$ & $0.338 * *$ & 37 & $0.441 * * *$ & - \\
\hline \multicolumn{7}{|l|}{ Stress difficulty: } \\
\hline - $\quad$ PIP-Communication & 57 & $0.284 * * *$ & $0.277 * * *$ & 37 & - & - \\
\hline - $\quad$ PIP- Emotional distress & 55 & - & $0.286^{* * *}$ & 35 & $0.354 * * *$ & - \\
\hline - $\quad$ PIP- Medical care & 56 & - & $0.278 * * *$ & 36 & $0.463 * * *$ & $0.365 * * *$ \\
\hline - $\quad$ PIP- Role function & 55 & - & - & 35 & $0.383 * * *$ & $0.355 * * *$ \\
\hline - $\quad$ PIP-Total & 56 & - & $0.268^{*}$ & 35 & $0.463 * *$ & $0.437 * *$ \\
\hline
\end{tabular}

Note: $* \mathrm{p}<0.05, * * \mathrm{p}<0.01, * * * \mathrm{p}<0.001$

Pearson correlation analysis was used to compute the association between HFS-P and PIP scores 
Table 4. Correlations between mothers', fathers' Hypoglycemia Fear Survey (HFS) total, Behavior subscale, and Worry subscale scores

\begin{tabular}{|c|c|c|c|c|c|c|}
\hline & & Father & & & Mother & \\
\hline & HFS-B & HFS-W & HFS-T & HFS-B & HFS-W & HFS-P total \\
\hline \multirow[t]{3}{*}{ HFS-B (Father) } & 1 & 0.041 & 0.55 & 0.378 & 0.287 & 0.393 \\
\hline & & 0.79 & 0.0001 & 0.011 & 0.059 & 0.008 \\
\hline & 45 & 44 & 44 & 44 & 44 & 44 \\
\hline \multirow[t]{3}{*}{ HFS-W (Father) } & & 1 & 0.857 & 0.081 & 0.207 & 0.201 \\
\hline & & & 0.0001 & 0.604 & 0.182 & 0.196 \\
\hline & & 44 & 44 & 43 & 43 & 43 \\
\hline \multirow[t]{3}{*}{ HFS-T (Father) } & & & 1 & 0.260 & 0.306 & 0.358 \\
\hline & & & & 0.092 & 0.046 & 0.018 \\
\hline & & & 44 & 43 & 43 & 43 \\
\hline \multirow[t]{3}{*}{ HFS-B (Mother) } & & & & 1 & 0.223 & 0.632 \\
\hline & & & & & 0.087 & 0 \\
\hline & & & & 60 & 60 & 60 \\
\hline \multirow[t]{3}{*}{ HFS-W (Mother) } & & & & & 1 & 0.896 \\
\hline & & & & & & 0 \\
\hline & & & & & 60 & 60 \\
\hline \multirow[t]{2}{*}{ HFS-P total (Mother) } & & & & & & 1 \\
\hline & & & & & & 60 \\
\hline
\end{tabular}

last 3 months, pediatric parenting stress or self-efficacy and children's glycemic control. Results found a negative correlation between SMBG and children's glycemic control $(\mathrm{r}=-0.3, \mathrm{p}=0.04)$.

Table 4 summarizes the correlations between mothers' and fathers' HFS-P total, Behavior subscale, and Worry subscale scores.

\section{Discussion}

In the current study, the HFS-P, PIP, and SED-P were used to measure worries and behaviors related to $\mathrm{FoH}$, parenting stress and self-efficacy (respectively) in parents of children with T1D in Iran. The Persian version of HFS-P, PIP and SED-P were found to be internally consistent and had good test-retest reliability. This finding suggests that these questionnaires are reliable and valid tools to assess the level of $\mathrm{FoH}$, stress and self-efficacy in a population with diabetes and could provide useful tools for identifying those families who may need diabetes education and support.

Cronbach's alpha for the Persian version of the HFS-P is comparable with the values reported by Gonder-Frederick et al. (7): 0.91 for the HFS-W, 0.76 for the HFS-B, 0.89 for the HFS-P total, and by Haugstvedt et al. (19): mothers 0.89 , fathers 0.89 for the HFS-W; mothers 0.69 , fathers 0.69 for the HFS-B and mothers 0.87 , fathers 0.84 for the HFS-P total. It should be noted that HFS-P demonstrated mostly good internal consistency in both subscales and total scores but the HFS-B subscale had the lowest internal consistency, which replicates previous findings and also reflects fewer items in this subscale ( 10 vs. 15 items) compared to the worry subscale $(7,19,23,24)$. In addition, the behavior subscale also includes items describing both appropriate (carry fast-acting sugar) and inappropriate actions (keep my child's blood sugar levels higher) to avoid hypoglycemia. While most previous studies, with only a few exceptions $(19,25)$, have focused on mothers, this study specifically examined both parents' FoH, stress, and self-efficacy in managing their child's diabetes. As it was reported previously (26), this study also found that mothers scored significantly higher on $\mathrm{FoH}$ than fathers, indicating that mothers have more hypoglycemic worry and perform more preventive behaviors to avoid hypoglycemia than fathers. An explanation could be that in Iran, as reported in some other studies (12), mothers are the primary caregivers and more often have the central role in managing the child's routine day-to-day diabetes care. A higher level of responsibility for child management is associated with increased parental $\mathrm{FoH}$ and emotional stress (5). Results showed no differences in the number of hypoglycemic episodes experienced by children with diabetes less than and longer than two years. But, in contrast to our expectation, higher mean HFS-B scores in mothers of children with diabetes for over 2 years indicate that they may engage in more actions to avoid hypoglycemia than mothers of children with diabetes for less than 2 years. Perhaps parallel to time passes; mothers could not cope with their child's condition which it seems a critical point. This finding may also remind us to focus on the ways to reduce $\mathrm{FoH}$ especially in mothers who have been dealing with diabetes longer in education programs, and we suggest that future interventions should target both the parental fear and proper ways to prevent hypoglycemia in children with T1D. Research suggests that, although fathers are usually not the primary caregiver of the child's diabetes care, their contribution to the family through insights, knowledge, adjustment, and behavior may be associated with disease management outcomes (12). Of note, the participation rate of fathers was less than mothers in the current study, and only 44 fathers completed all of the items on the HFS-P (one father completed only the Behavior subscale), 35-39 fathers completed all PIP questions, and 42 fathers completed all SED-P questions. By their report, fathers did not answer some of the questions because they had no knowledge of the information being assessed since the mothers were typically responsible for childcare in that situation. While results of this study indicate that mothers are more involved in their child management and have more FoH, fathers' mean HFS-B scores correlated with children's total insulin dose per day, which may reflect the higher risk for hypoglycemia with higher insulin doses. Thus it may be that the psychological impact 
of children's diabetes is different between mothers and fathers and as Mitchell et al. recommended (16), future research is recommended with a broader range of factors impacted by child health that may contribute to fathers' psychological health.

Cronbach's alpha for the Persian version of the PIP and SED-P are comparable with the values reported by Streisand et al. (5): $\mathrm{PIP}-\mathrm{F}=0.94$ and PIP-D $=0.95$; by Grey et al (21): $\mathrm{SED}-\mathrm{P}=0.88$ for adolescents and by Streisand et al. (5): $\mathrm{SED}-\mathrm{P}=0.89$ for parents, respectively. PIP findings also suggest that half of the mothers and nearly $40 \%$ of fathers of children with T1D are experiencing considerable parenting stress and symptomatic emotional distress. As reported previously (25), we also found that parents' FoH was positively correlated with increased frequency and difficulty of pediatric parenting stress. HFS-W subscale and HFS-P total scores correlated significantly with measures of parenting stress. These findings support the validity of the Iranian version of HFS-P and furthermore the association between a higher level of $\mathrm{FoH}$ and parental stress emphasizes the need for programs to support and guide both parents. It is very important for diabetes educators to recognize high $\mathrm{FoH}$ in parents (27). Although some level of fear is normal and could motivate parents to protect their children from the risk of hypoglycemic episodes, extreme FoH might lead to poorer coping strategies, such as overeating to prevent hypoglycemia, administering lower doses of insulin and over-feeding children without insulin injection (4). Patton et al. reported a correlation between higher blood glucose concentration and parental behavior aiming to avoid hypoglycemia, whereas the Norwegian study found a positive correlation between the HFS-W and HbA1c $(19,23)$. Our data showed that many children $(91.7 \%)$ had suboptimal glycemic control, which could place them at high risk of developing microvascular complications, but did not find any association between parental FoH and glycemic control (HbAlc) maybe because of limited sample size.

None of the FoH, self-efficacy and the parenting stress measures correlated with the number of hypoglycemic episodes in the last 3 months. Perhaps an association would have been identified with a more detailed assessment of hypoglycemia episodes in last 3 months like that conducted in studies by Haugstvedt et al. (19) and Patton et al. (23). Others have found that $\mathrm{FoH}$ is related to the number of episodes associated with unconsciousness or seizure in the child (18).

Despite the noted findings, children's glycemic control was negatively correlated with SMBG. This finding replicates the important association reported by Ziegler et al. (28) in a large database study that emphasize the importance of SMBG in better glycemic control again. So the frequency and timing of SMBG should be addressed clearly in diabetes education courses for both parents.

Some limitations of our study are the cross-sectional design, which makes it impossible to explore the causal association between variables and self-report measures, which are vulnerable to a reporting bias. Although we followed suggested scientific procedures when translating HFS-P,
PIP, and SED-P into Persian and all of them have demonstrated acceptable reliability and validity in our study, some changes may still be needed based on country culture. For example, only $25 \%$ of mothers in this study were employed, and there is a PIP question about "Being unable to go to work". In addition, questions in HFS-P measure both inappropriate and appropriate behaviors to prevent hypoglycemia, which can be another problem. It would be helpful for researchers using various translations of the same questionnaires to share their cross-cultural experiences in a collaborative manner in order to prepare a culturally comprehensive manual. The sample size was limited, and this could be the reason for the statistically non-significant associations identified between the children's HbAlc and parents' FoH, stress and self-efficacy.

\section{Conclusion}

In conclusion, the results of this study indicate that the Persian version of the HFS-P, PIP, and SED-P are psychometrically valid and reliable instruments to measure $\mathrm{FoH}$, parenting stress and self-efficacy in managing diabetes in this target group, but may need some cultural changes. Moreover, from the clinical and research perspectives, these questionnaires could be used for identifying those families who are at risk and may need education; and could provide a useful tool for assessing the effect of interventions (27). Also, findings indicate that difficulties in parents' (especially mothers') level of high fear of their child's hypoglycemia correlated with increased frequency and difficulty of parental stress. So diabetes education and counseling, problem-solving and stress management training for parents could prepare them to manage their child diabetes. Based on findings from the current study, important implications for diabetes education are: considering psychological adjustment, identifying diabetes-related fear and stress in parents of children with T1D, encouraging fathers to become more involved in the child's diabetes management to reduce mothers feeling overstressed. Because improving health outcomes of children with diabetes is a multifaceted process, the results of this study suggest that targeting parental FoH, stress, and self-efficacy may be helpful components of structured education courses in this population.

\section{Acknowledgments}

We would wish to thank the parents and children who graciously agreed to participate in this research as well as the president and members of the Gabric Diabetes Education Association. The authors also thank Dr. Randi Streisand, Dr. Gary M. Ingersoll, Dr. Leann L. Birch and Dr. Susana R. Patton for sending questionnaires and their expert suggestions. This study was funded by Vice Chancellor of Research, Iran University of Medical Sciences (MT/534).

\section{Conflicts of Interest}

The authors have no conflict of interest. 


\section{References}

1. Zayed H. Genetic Epidemiology of Type 1 Diabetes in the 22 Arab Countries. Curr Diab Rep. 2016;16(5):37.

2. Chuang LM, Tsai ST, Huang BY, Tai TY. The status of diabetes control in Asia--a cross-sectional survey of 24317 patients with diabetes mellitus in 1998. Diabet Med. 2002;19(12):978-85.

3. Walsh MG, Zgibor J, Borch-Johnsen K, Orchard TJ. A multinational comparison of complications assessment in type 1 diabetes: the DiaMond substudy of complications (DiaComp) level 2. Diabetes Care. 2004;27(7):1610-7.

4. Delamater AM. Psychological care of children and adolescents with diabetes. Pediatr diabetes. 2009;10 Suppl 12:175-84.

5. Streisand R, Swift E, Wickmark T, Chen R, Holmes CS. Pediatric parenting stress among parents of children with type 1 diabetes: the role of self-efficacy, responsibility, and fear. J Pediatr Psychol. 2005;30(6):513-21.

6. Wild D, von Maltzahn R, Brohan E, Christensen T, Clauson P, Gonder-Frederick L. A critical review of the literature on fear of hypoglycemia in diabetes: Implications for diabetes management and patient education. Patient Educ Counsel. 2007;68(1):10-5.

7. Gonder-Frederick LA, Fisher CD, Ritterband LM, Cox DJ, Hou L, DasGupta AA, et al. Predictors of fear of hypoglycemia in adolescents with type 1 diabetes and their parents. Pediatr Diabetes. 2006;7(4):215-22.

8. Gonder-Frederick L. Fear of hypoglycemia: A review. Diabetic Hypoglyc. 2013;5(3):3-11.

9. Delamater AM. Psychological care of children and adolescents with diabetes. Pediatr Diabetes. 2007;8(5):340-8.

10. Bulsara MK, Holman CD, Davis EA, Jones TW. The impact of a decade of changing treatment on rates of severe hypoglycemia in a population-based cohort of children with type 1 diabetes. Diabetes care. 2004;27(10):2293-8.

11. Streisand R, Braniecki S, Tercyak KP, Kazak AE. Childhood illnessrelated parenting stress: the pediatric inventory for parents. J Pediatr Psychol. 2001;26(3):155-62.

12. Dashiff C, Morrison S, Rowe J. Fathers of children and adolescents with diabetes: what do we know? J Pediatr Nurs. 2008;23(2):101-19.

13. Phares V, Lopez E, Fields S, Kamboukos D, Duhig AM. Are fathers involved in pediatric psychology research and treatment? J Pediatr Psychol. 2005;30(8):631-43.

14. Landolt MA, Vollrath M, Laimbacher J, Gnehm HE, Sennhauser FH. Prospective study of posttraumatic stress disorder in parents of children with newly diagnosed type 1 diabetes. J Am Acad Child Adolesc Psychiatry. 2005;44(7):682-9.

15. Grus CL, Lopez-Hernandez C, Delamater A, Appelgate B, Brito A, Wurm G, et al. Parental self-efficacy and morbidity in pediatric asthma. J Asthma. 2001;38(1):99-106.

16. Mitchell SJ, Hilliard ME, Mednick L, Henderson C, Cogen FR, Streisand R. Stress among fathers of young children with type 1 diabetes. Fam Syst Health. 2009;27(4):314-24.

17. McMahon SK, Airey FL, Marangou DA, McElwee KJ, Carne CL, Clarey AJ, et al. Insulin pump therapy in children and adolescents: improvements in key parameters of diabetes management including quality of life. Diabet Med. 2005;22(1):92-6.

18. Clarke WL, Gonder-Frederick A, Snyder AL, Cox DJ. Maternal fear of hypoglycemia in their children with insulin dependent diabetes mellitus. J Pediatr Endocrinol Metab. 1998;11 Suppl 1:189-94.

19. Haugstvedt A, Wentzel-Larsen T, Graue M, Sovik O, Rokne B. Fear of hypoglycaemia in mothers and fathers of children with Type 1 diabetes is associated with poor glycaemic control and parental emotional distress: a population-based study. Diabet Med. 2010;27(1):72-8

20. Grossman HY, Brink S, Hauser ST. Self-efficacy in adolescent girls and boys with insulin-dependent diabetes mellitus. Diabetes care. 1987;10(3):324-9.

21. Grey M, Davidson M, Boland EA, Tamborlane WV. Clinical and psychosocial factors associated with achievement of treatment goals in adolescents with diabetes mellitus. J Adolesc Health. 2001;28(5):377-85.

22. Sonne DP, Hemmingsen B. Standards of medical care in diabetes2017. diabetes care 2017;40(Suppl. 1):S1-S135. Diabetes Care. 2017;40(7):e92-e3.
23. Patton SR, Dolan LM, Henry R, Powers SW. Parental fear of hypoglycemia: young children treated with continuous subcutaneous insulin infusion. Pediatr Diabetes. 2007;8(6):362-8.

24. Gonder-Frederick LA, Schmidt KM, Vajda KA, Greear ML, Singh $\mathrm{H}$, Shepard JA, et al. Psychometric properties of the hypoglycemia fear survey-ii for adults with type 1 diabetes. Diabetes Care. 2011;34(4):801-6.

25. Viaene AS, Van Daele T, Bleys D, Faust K, Massa GG. Fear of Hypoglycemia, Parenting Stress, and Metabolic Control for Children with Type 1 Diabetes and Their Parents. J Clin Psychol Med Sett. 2017;24(1):74-81.

26. Haugstvedt A, Wentzel-Larsen T, Aarflot M, Rokne B, Graue M. Assessing fear of hypoglycemia in a population-based study among parents of children with type 1 diabetes - psychometric properties of the hypoglycemia fear survey - parent version. BMC Endocrine Disord. 2015;15(1).

27. Gonder-Frederick L, Nyer M, Shepard JA, Vajda K, Clarke W. Assessing fear of hypoglycemia in children with Type 1 diabetes and their parents. Diabetes Manag (Lond). 2011;1(6):627-39.

28. Ziegler R, Heidtmann B, Hilgard D, Hofer S, Rosenbauer J, Holl R. Frequency of SMBG correlates with $\mathrm{HbA1c}$ and acute complications in children and adolescents with type 1 diabetes. Pediatrs Diabetes. 2011;12(1):11-7. 